\title{
Research on Color Application in Modern Design
}

\author{
Wenjun Han \\ Huanghe Science and Technology College \\ Zhengzhou, China
}

\begin{abstract}
Design without color lacks vitality. Therefore, color has become a kind of mainstream in modern design, especially that research emphasizing on psychological color and engineering color makes color design the mainstream setting fashion. "Micro-color's" positive energy is also the trend of modern design. In fact, modern color design has become comprehensive science combining color optics, color physiology, color psychology, color aesthetics and color engineering. So it is undoubted that there is important practical significance in mastering application and research of color in modern design.
\end{abstract}

Keywords—modern design; color application; micro-color

\section{INTRODUCTION}

Color is the first silent language in modern design. In terms of color application, modern design has transferred from oneway color show to concept innovation of systematic color. As we all know, to any design, color is the primary element entering people's first impression. It is self-evident that application of color plays an important role in modern design. Then, new reform of color in modern design is mainly displayed in the following several points.

\section{MODERN DESIGN EMPHASIZING ON RESEARCH ON MENTALITY, COLOR ENGINEERING AND SO ON}

Psychologist's research shows that people's first feeling is vision and color influences vision most. People's behavior is influenced by color because people's behavior is easily governed by emotion at so many times. Since color derives from congenital color in nature, like blue sky, bright red blood, golden sun and so on, color can influence people's mental state and state of mind. When seeing these colors the same as congenital colors of nature, people can naturally associate with relevant feeling experience of these natural objects. This is the most primitive influence. It is also probably due to that people in different regions, countries, nationalities and personalities have common feeling experience of some colors. For example, red usually brings these feelings to people: excited, passionate, active, bold and powerful, and solemn, respectful, beaming, happy and so on. Green is the color of prairie and forest in nature and has the meaning of forever vitality, ideal, youth, safety, fresh and peace, giving people cold and refreshing feelings. Blue makes people feel distant, silent, void and so on. With social development, there are more and more objects coming to people's mind due to influence of color and people's feeling about color gets more and more complicated.

Color influences modern people's social life, therefore modern design has color psychology. Objectively speaking, color is a kind of stimulus and symbol to people. Subjectively speaking, color is a kind of reaction and behavior. Color psychology penetrates through vision, consciousness, sentiment, memory, thought, will, symbol and so on in sequence. Application of color pays great attention to this kind of cause-and-effect relationship, namely transforming into psychological standard of color from accumulation of color experience and reaction to each stimulus.

In addition, modern design also emphasizes on color engineering, including color notation, colorimetry method, color scheme design, color conditioning, color management and so on. For example, packaging chromatics is specific application of packaging color design, color reproduction and so on and is organic unification of natural color, social color and artistic color. Packaging chromatics starts from packaging color and systematically reflects color formation and expression, and the phenomenon and law of color design and reproduction. It is organic unification of color composition, colorimetry, printing chromatics and other relevant contents and it is organic combination of perceptual knowledge and rational analysis of packaging color.

\section{COLOR DESIGN SETS FASHION MAINSTREAM}

Color specialists think that fashion color has personality and age meaning. It can arouse strong resonance in people's mind. This kind of strong vision attraction creates lifeful fashion culture. Prevailing regularity of fashion color usually is that people are sick for green and orange after long prevailing of red and blue; people are sick for medium and deep color after long prevailing of light and shallow color; people pursue cool color after long prevailing of bright color; and people are sick for color after long prevailing of warm color. Probably this is people's physiology and ecology balance of appreciating demand which is formed due to physiological need.

In setting fashion, modern color design especially emphasizes on seven color factors: The first is cold and warm color: Red, orange and yellow usually make people think of rising sun and burning fire, boasting warm feeling. Blue cyan usually makes people think of ocean, clear sky and shallow, boasting cold feeling. Red, orange and yellow bring people warm feeling while blue and green bring people cold feeling. Difference in cold and warm color is also related to lightness and purity. Color with high lightness usually has cold feeling while color with low lightness usually has warm feeling. Color with high purity usually has warm feeling while color with low purity usually has cold feeling. In neutral colors, white has 
cold feeling, black has warm feeling and gray is medium. The second is color's light and heavy feeling: It is usually determined by lightness. High lightness has light feeling while low lightness has heavy feeling. White is the lightest while black is the heaviest. Color matching with low lightness has heavy feeling while color matching with high lightness has light feeling. The third is color's soft and hard feeling: It is related to lightness and purity. Gray color with high lightness has soft feeling while gray color with low lightness has hard feeling. The higher the purity is, the harder it feels. The lower the purity is, the softer it feels. Strong contrast color has hard feeling while weak contrast color has soft feeling. The fourth is color's strong and weak feeling: High purity color has strong feeling while low purity color has weak feeling. Chromatic color has stronger feeling than neutral color. Among chromatic colors, red is the strongest. Big contrast color has strong feeling while low contrast color has weak feeling. Namely, it feels strong with dark background and bright painting or bright background and dark painting. It feels weak with dark background and painting or bright background and painting. The fifth is color's vivid sense and depression: They are related to purity. Bright color with high lightness has vivid sense while dark and muddy color has depression. Lowly bright color is easy to generate depression while highly bright color is easy to generate vivid sense. Strong contrast color has vivid sense while weak contrast color has depression. The sixth is color's excitement and quiet sense: They are related to hue, lightness and purity. Among them, purity's effect is the most obvious. In terms of hue, warm colors of red and orange have excitement while cold colors of blue and green have quiet sense. In terms of lightness, color with high lightness has excitement while color with low lightness has quiet sense. In terms of purity, high purity color has excitement while low purity color has quiet sense. Therefore, among warm colors, the color with highest lightness and purity has the strongest excitement. Among cold colors, the color with lowest lightness and purity has the quietest sense. Strong contrast color has excitement while weak contrast color has quiet sense. The seventh is color's magnificence and simplicity: They are mostly related to purity and secondarily related to lightness. Bright and fresh color has magnificence while muddy and dark color has simplicity. Chromatic color has magnificence while neutral color has simplicity. Match colors with application of hue contrast have magnificence. Among them, complementary color is the most gorgeous. Strong contrast color has magnificence while weak contrast color has simplicity.

In addition, modern design emphasizes on color matching, mainly displayed in: Intermedium color of hale color is red, intermedium color of happy color is yellow and orange, intermedium color of elegant color is pink, intermedium color of gentle color is soft rose hermosa, intermedium color of graceful color is rose and light purple, and intermedium color of gorgeous color is tangerine, blue and gold. This kind of technique of expression is jump of space color. Application of apple green, deep blue, bright red, pure yellow and other high purity colors in great quantity is bold and flexible. It is not only following modern design style, but also show of personality. In terms of emphasizing on functionality, modern design has strong color contrast and gives people unconstrained feeling of vanguard.

\section{IV. "MICRO-COLOR” IS FASHION TREND OF MODERN DESIGN}

"Micro-color" will become development direction of modern design. It emphasizes on combination of natural color and human color, sense of harmony and low-carbon life style of saving energy and environmental protection. In concise and direct decoration design pressing close to nature, there is no pattern decoration, but only line and color plate. It emphasizes on perfect combination of simple structure and comfortable function. Harmony of color matching and line gives out different artistic magic. As to black and white, romantic hue, simple atmosphere and bolster's color ornament give out passion and vitality of life. Coordinated and symmetrical techniques of red and white make arrangement of each detail showing comfortable atmosphere.

"Micro-color" emphasizes greatly on integration of natural color element in modern design. No matter it is furniture, costume or transportation tool, their material and color respect features of natural material before conducting matching. "Micro-color" emphasizes on scientific combination of human and nature and environment with concentrated embodiment of the concept of green design and environmental protection design. Black and white is the most commonly used colors and they can go with some abundant colors to show artistic magic. In overall design, it emphasizes on function and simplifies design with concise lines, making people feel the most comfortable atmosphere of "micro-color".

Originally, nature and human society is a colorful, brilliant and varied world, in which different kinds of things exist in different colors. With the help of color, people observe different kinds of objects from the outside to the inside and from the shallower to the deeper. It is an important method for human to know the world. It is an important reform of color design by people to introduce modern scientific means to modern design color research. People's recognition of color is a comprehensive process, including physical phenomenon, psychological sensing and psychological effect. If we extend it to application of color, it also includes color design and color engineering. Therefore, modern color design is comprehensive science combining color optics, color physiology, color psychology, color aesthetics and color engineering, covering extremely wide areas.

Before appearance of fashion color, people use traditional color and common color. With people's imitation and pursuit of changes, new meaning and fashion color, fashion color becomes a conception to spread. In fact, fashion trend of color can be known, forecast and guided, including social trend of thought, economic situation, living environment, psychological changes, consumption tendency and so on. All these influence trend of fashion color in different degrees. Fashion color is different from traditional color or advanced color. It is required that fashion color should be innovative and can be understood and accepted by most people, being unique and not strange. To some degree, development trend of fashion color is also influenced by the law of color itself, such as trend of color changes, color combination proportion, color sequencing and so on. All these are influenced by law of color itself. 


\section{CONCLUSION}

Color's influence on modern design has extremely important significance. Proper usage of color design can relieve fatigue, improve work efficiency and reduce accidents in work, can create comfortable environment and increase the joy of life in life and even can be used to treat an illness medically. No matter it is architecture or costume design, usage of warm colors can increase warm feeling. In traffic signals, strong matching of red and green, yellow and blue, and black and white can arouse attention easily, thus avoiding accidents. If used in advertisement, it can catch the attention to realize the effect of publicity. Cargo box can be painted in pink to relieve bearer's mental weight burden. Residence uses bright matching colors to give people roomy and comfortable feeling. Entertainment venues use gorgeous and exciting colors to enhance happy, joyous and passionate atmosphere. School and hospital use bright and clear matching colors to create a quiet, clean, healthy and peaceful environment for students and patients. Summer clothes use cold color while winter clothes use warm color to adjust cold and warm feeling. Children's clothes use strong, jumping, twinkling and lively matching colors to express their vivaciousness better and win people's love. Beautiful and brilliant eye ornament hue can make women appear young, bold, lively and vigorous. Plain, profuse and calm costume hue can set off young men's steady, confident and mature character. If young men wear bright red and bright green clothes, they will give people frivolous and unstable feeling. Because putting their energy entirely into career people lack energy to pursue color stimulation. Medically, light blue can bring down a fever and lower blood pressure, chocolate can enhance blood pressure and increase metabolism, blue fluorescent lamp shining on infant with jaundice has certain effect, green can help patients rest, red and orange can enhance appetite and purple can make pregnant women calm down and reduce pain of delivery.

\section{REFERENCES}

[1] Chen Liannian. Color Design. Southwest China Normal University Press.

[2] (American) Sean Adams, Morioka and Stone. Color Application: Classic Originality of Color Matching in Graphic Design. Translated by Yu Yang. Beijing: China Youth Press, 2007.

[3] (Japanese) Designing Editorial Department. Color Design(Japanese Graphic Designer Reference Manual). Compiled by Zhou Yanhua and Hao Wei. Beijing: Posts and Telecom Press, 2011.

[4] (Korean) Jin Rongshu. Color Psychology in Design. Translated by Wu Chuanhai and Cao Ting. Beijing: Posts and Telecom Press, 2011.

[5] Wu Zhenbao and Zhang Wencai. Color Theory and Application. Jiangsu Fine Arts Press

[6] Wu Fan. Color Design. Shanghai: Shanghai Jiaotong University Press, 2012.

[7] Lu Hong and Yu Xianglong. Modern Design Element: Color Design. Nanning: Guangxi Fine Arts Press, 2006. 